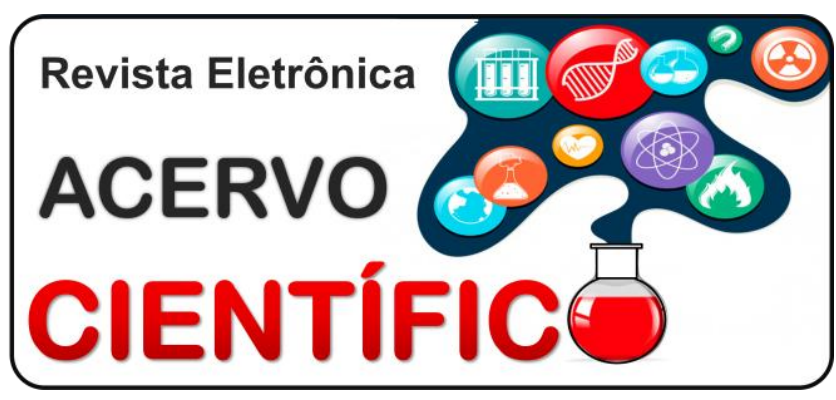

\section{REVISÃO BIBLIOGRÁFICA}

Recebido em: 9/2019

Aceito em: 9/2019

Publicado em: 10/2019

\title{
Acessibilidade para idosos em ambientes internos: a atualidade dos projetos no design de interiores
}

\author{
Accessibility for elderly in indoor environments: current design in interior design
}

\author{
Accesibilidad para personas mayores en entornos interiores: diseño actual en diseño \\ interior
}

Adilson Mendes de Figueiredo Júnior ${ }^{1 *}$, Eliana do Socorro Monteiro dos Santos ${ }^{1}$, Letícia Martins Monteiro $^{1}$, Elizangela Fonseca de Mendonça ${ }^{1}$, Herlani Cristina Oliveira Barreto ${ }^{1}$, Willame Renato Lima de Siqueira ${ }^{1}$, Gabriel Santos de Lima ${ }^{1}$, Elenice Melo Hughes de Figueiredo ${ }^{2}$, Carla Caroline Lobo de Souza ${ }^{3}$, Braydson Nunes dos Santos ${ }^{3}$.

\begin{abstract}
Resumo: Esse artigo buscou identificar como ocorre, atualmente, a acessibilidade para idosos em ambientes internos, considerando-se projetos no design de interiores. Trata-se de um estudo de natureza qualitativo-descritiva - o qual quantificou e descreveu os achados científicos - sob método de revisão bibliográfica. Respeitou-se a totalidade dos achados científicos e organizaram-se tabelas caracterizadoras para melhor observação e entendimento de cada artigo científico. A ausência de critérios técnicos e de equipamentos que tornem um ambiente acessível, tanto interno quanto social ainda produz dificuldades no critério acessibilidade. A avaliação do ambiente que envolve as relações diárias envolvendo idosos se tornou um fator de importância exponencial para manutenção da saúde desses indivíduos e da autonomia das atividades domésticas. Conclui-se ser essencial, minimizar as "barreiras" internas do lar de idosos em relação à acessibilidade e à funcionalidade do espaço.
\end{abstract}

Palavras-chave: Acessibilidade, Idoso, Arquitetura.

\begin{abstract}
This article sought to identify how accessibility is currently available for seniors indoors, considering interior design projects. This is a qualitative-descriptive study - which quantified and described the scientific findings - under a literature review method. All scientific findings were respected and characterizing tables were organized for better observation and understanding of each scientific article. The absence of technical criteria and equipment that make an accessible environment, both internal and social, still produces difficulties in the accessibility criterion. The assessment of the environment that involves daily relationships involving the elderly has become a factor of exponential importance for maintaining the health of these individuals and the autonomy of domestic activities. It is concluded that it is essential to minimize the internal "barriers" of the nursing home regarding accessibility and functionality of the space.
\end{abstract}

Keywords: Accessibility, Old man, Architecture.

\footnotetext{
1 Escola Superior da Amazônia (ESAMAZ), Belém-Pará. *E-mail: adilsonmdfj@hotmail.com

2 Universidade da Amazônia (UNAMA), Belém-Pará.

3 Universidade do Estado do Pará (UEPA), Belém-Pará.
} 
Resumen: Este artículo buscaba identificar cómo la accesibilidad está actualmente disponible para personas mayores en interiores, considerando proyectos de diseño de interiores. Este es un estudio cualitativo descriptivo, que cuantificó y describió los hallazgos científicos, bajo un método de revisión de literatura. Se respetaron todos los hallazgos científicos y se organizaron tablas de caracterización para una mejor observación y comprensión de cada artículo científico. La ausencia de criterios técnicos y equipos que hagan un entorno accesible, tanto interno como social, aún produce dificultades en el criterio de accesibilidad. La evaluación del entorno que involucra las relaciones diarias con los ancianos se ha convertido en un factor de importancia exponencial para mantener la salud de estos individuos y la autonomía de las actividades domésticas. Se concluye que es esencial minimizar las "barreras" internas del hogar de ancianos con respecto a la accesibilidad y funcionalidad del espacio.

Palabras clave: Accesibilidad, Ciudadano mayor, Arquitectura.

\section{INTRODUÇÃO}

A avaliação do ambiente que envolve as relações diárias envolvendo idosos se tornou um fator de importância exponencial para manutenção da saúde desses indivíduos e da autonomia das atividades domésticas. O Brasil, assim como diversos outros países, passa por um crescimento populacional que projeta a população para um aumento exponencial de faixa etária. Nos últimos 20 anos a população idosa brasileira praticamente duplicou e entre as variáveis que justificam esse dado pode-se citar a diminuição da fecundidade nos últimos anos e a redução da mortalidade infantil. Porém, percebe-se que junto ao novo cenário populacional ainda é visível a deficiência na maioria dos ambientes onde os idosos estão inseridos, o que não permite a adequação entre o individuo e o meio em que vivem, tal fato relaciona-se a diversos riscos que geram acidentes domésticos e que reduzem a melhoria das atividades cotidianas da população em questão (SILVA NM et al, 2015).

O processo do "envelhecer" está envolvido com muitos desafios, pois o aumento da expectativa de vida gera preocupação, principalmente, em relação à qualidade de vida e o bem-estar dos idosos, principalmente, no que se refere à acessibilidade e respeito às individualidades. Dessa forma, é de fundamental importância, identificar os fatores de risco de quedas para que se possam planejar estratégias de prevenção, reorganização ambiental e de reabilitação funcional e para capacitação profissional na perspectiva de adequação do meio de vivência desses idosos. As condições físicas, onde os idosos residem, devem promover acessibilidade, com o intuito de diminuir esses riscos e promoverem saúde e bem-estar (GARBIN CAS et al, 2015).

Junto ao crescimento das cidades e o processo de urbanização, a busca por atividades de lazer e da naturalidade e individualidade das atividades domésticas é cada vez mais crescente. O lar ainda se constitui com um dos principais espaços utilizados pelos idosos. No entanto, em decorrência do processo de envelhecimento, os idosos enquadram-se no grupo de pessoas com mobilidade reduzida, o que significa dizer que apresentam redução da mobilidade, flexibilidade, coordenação motora e percepção. Pela dada questão, a observação e integralidade das características como luminosidade, estrutura física entre outras são determinantes para a adaptação do indivíduo ao ambiente (LUIZ FI e TEIXEIRA JC, 2016).

É necessário perceber que para a concretização da inclusão das pessoas idosas na sociedade e nas projeções dos trabalhos em design de interiores é necessário que essas disponham de garantias de atendimento individualizado e de condições de utilizar de forma plena os ambientes, objetos e serviços necessários à sua existência, com autonomia, independência e segurança. Acessibilidade e acolhimento devem representar as ferramentas, constantemente presentes, na formação dos novos ambientes de convivência e que possam servir como modificadores dos espaços não acessíveis e/ou não ergonômicos (SANTOS MIPO et al, 2016).

O envelhecimento humano vivenciado pela população brasileira nos dias atuais pode ser explicado pelos progressos tecnológicos e melhorias nos padrões de saúde da população, com aumento significativo da 
expectativa de vida, porém as ações de inclusão social e adaptação do meio aos indivíduos inseridos nesse processo ainda passam por um período de maturação e necessita alcançar níveis mais dinâmicos e presentes, visto a velocidade de mudanças do novo padrão etário da sociedade brasileira (AUSTREGÉSILO SC et al, 2015).

A atual pesquisa teve o objetivo de revisar na literatura acerca da identificação de como ocorre, atualmente, a acessibilidade para idosos em ambientes internos, considerando-se projetos no design de interiores.

\section{MÉTODOS}

Trata-se de um estudo de natureza qualitativo-descritiva - o qual quantificou e descreveu os achados científicos - sob método de revisão bibliográfica desenvolvida por meio da Revisão Integrativa da Literatura (RIL). A busca de dados ocorreu pelo processo de Revisão Integrativa de Literatura que foi realizada em 6 (seis) etapas distintas, sequenciais e que se interligam: Definição da pergunta norteadora; 2) Busca da literatura; 3) Coleta de dados; 4) Análise crítica dos estudos incluídos; 5) Discussão dos resultados; 6) Apresentação da Revisão integrativa. Como fase inicial formulou-se a seguinte questão: Como ocorre, atualmente, a acessibilidade para idosos em ambientes internos, considerando-se projetos no design de interiores?

Tal pergunta norteou a busca bibliográfica associada a todos os critérios de filtragem dos artigos, ou seja, utilizaram-se apenas os achados científicos que possuíam coerência com o objeto do estudo. A busca foi realizada em banco de dados eletrônicos na internet. Foram utilizados na busca de descritores: acessibilidade, idosos e arquitetura.

Os artigos foram selecionados e analisados, de acordo com o tema proposto. Após a seleção dos artigos indexados, foi realizada uma avaliação e análise do material obtido, para selecionar o que era de interesse para a pesquisa e eliminar os artigos repetidos ou aqueles que fugissem do objeto do estudo, em seguida realizou-se uma leitura minuciosa e pontual, a fim de não serem perdidos aspectos importantes para o enriquecimento de estudo e constituição do quadro de caracterização dos resultados da pesquisa.

Como benefícios espera-se que o atual estudo possa servir de base para novas diretrizes e programas de atualização profissional, visto a importância do trabalho envolvendo a dinâmica profissional dentro do contexto de prevenção de acidentes domésticos e até mesmo para a melhoria das condições de interação entre o individuo e o ambiente físico.

Os critérios de inclusão adotados foram artigos científicos completos nacionais e que envolvessem a acessibilidade para idosos, como critérios de exclusão, foram excluídos artigos que associavam acessibilidade com idosos com adoecimento prévio e artigos com mais de 10 anos.

\section{RESULTADOS E DISCUSSÕES}

O crescimento etário nacional constitui uma nova transição demográfica em diversos estados do país e até mesmo em outros continentes. Critérios de acessibilidade e funcionalidade ainda permitem a compreensão de como ocorre a inclusão sócio espacial da população, principalmente, grupos com determinadas limitações, como a população idosa. Identificar e minimizar os problemas relacionados à mobilidade e acessibilidade no interior das residências colabora para a preservação da independência e segurança do morador, reforçando o direito primordial de livre arbítrio e de ir e vir. Muitos espaços ainda apresentam a ausência de critérios técnicos e equipamentos que suportem condições acessíveis, tanto em edificações quanto em outros ambientes, tal fato, promove uma "barreira" nas atividades cotidianas exercida pelos indivíduos (SILVA NM et al, 2015).

A avaliação do critério acessibilidade no interior das residências constitui uma ação primordial para o bem-estar físico, biológico e mental, pois segundo a Sociedade Brasileira de Geriatria e Gerontologia, a queda está em primeiro lugar do ranking de causa de acidentes em pessoas com mais de 60 anos, os 
acidentes ali presentes podem causar sequelas tanto físicas como psicológicas e que muitas vezes podem ser irreversíveis e levar ao óbito. O processo de alterações fisiológicas e patológicas é diretamente proporcional ao crescimento etário e, dessa forma ocorre enfraquecimento natural dos ossos e músculos, alterações visuais, desatenção e perda de equilíbrio e alterações no padrão da marcha, mas tão preocupante quanto às alterações biológicas são as alterações presentes em um ambiente que 0 torna inseguro (TOWATA TM, 2014).

O envelhecimento biológico causa, na grande maioria das vezes, alterações significativas no sistema neurológico, musculoesquelético e cardiovascular, tais alterações refletem sobre a acuidade visual, o equilíbrio e a locomoção, limitando os indivíduos idosos em sua autonomia e segurança, principalmente quando apresentam doenças crônicas degenerativas. Há uma necessidade de projetar ambientes que possam minimizar as alterações funcionais e fisiológicas no idoso, principalmente em relação aos espaços e utensílios domésticos, facilitando as ações do dia a dia e projetando o idoso para um cotidiano seguro e funcional (FERNANDES JCFA e CARVALHO RJM, 2011).

A reorganização dos serviços de saúde e de adaptação social não se baseia apenas no reconhecimento das políticas públicas ou serviços oferecidos, mas principalmente, na concepção dos profissionais envolvidos. Com a intenção de equiparação das oportunidades de adaptação e acessibilidade às pessoas idosas é necessária uma abordagem de diversos fatores integrados como família, sociedade e saúde. A partir do momento que se reconhece as diversas necessidades e se promove o respeito, as ações tornamse mais dinâmicas e presenciais, favorecendo a inserção do idoso em sua residência (GIRONDI JBR e SANTOS SMA, 2011).

O reconhecimento do nível de acessibilidade das diferentes áreas que constituem a residência de um idoso é de fundamental importância para a estrutura e elaboração de projetos que buscam acessibilidade, proporcionar a independência e autonomia da população idosa ainda significa um meio de se evitar acidentes por quedas e projeta o indivíduo a realizar suas atividades de maneira mais concreta (ALMEIDA RLS et al, 2015). As modificações das residências necessitam, por várias vezes, passarem por ajustes para que a acessibilidade se torne algo presente na vida dos idosos, porém a arquitetura associada ao design de interiores ainda necessita se adequar às necessidades da população em questão e conhecer as peculiaridades e as atividades diárias exercidas pelo mesmo, dessa maneira, é possível entender que o envelhecimento é um processo real e que necessita ser respeitado para que as atividades do cotidiano possam ser atos mais seguros e prazerosos (NEVES FC e BIFANO ACS, 2015).

A identificação das interfaces do ambiente residencial pode apresentar propriedades positivas ou não para a vivência do idoso, a visão multidisciplinar do profissional do design de interiores tem o intuito de compreender a complexidade da vida humana, que hoje sendo mais ativa, cria uma nova dinâmica no interior das residências. É pertinente identificar os aspectos relativos a todos os fatores que interferem na acessibilidade de um espaço planejado para que o mesmo não ofereça riscos para os indivíduos através da identificação dos fatores negativos que significa um olhar diferenciado onde o profissional possa não só visualizar uma atual situação, mas se ver presente dentro de uma dinâmica que será presencial a todos os indivíduos (SILVA NM et al, 2015).

De acordo com Towata TM (2014), a queda não é um fator relacionado apenas às alterações biológicas, como os problemas da visão, mas o envelhecimento pode trazer inúmeras situações no cotidiano que podem ser acrescentadas de um risco exponencial devido à estruturação de pisos, paredes e até mesmo a disposição de móveis dentro do ambiente residencial. O ambiente doméstico necessita ser avaliado e estudado, principalmente, para a observação de elementos considerados inseguros dentro dos projetos de design de interiores, como pavimentos irregulares, revestimento impróprio e etc.

Os estudos de Júnior RC et al (2013) corroboram os estudos de Towata TM (2014) e afirmam que a preocupação em relação ao planejamento de um ambiente construído e a sua sensibilidade ainda representa uma ação de bastante relevância considerando que os temas emergentes relacionados ao estudo da velhice e envelhecimento significa uma mudança no modelo que nós temos de estruturação dentro de residência. A tarefa de projetar um ambiente para usuários idosos infelizmente ainda é tratada de 
forma superficial e muitas vezes são comparadas as estratégias de pessoas com deficiência física, a diminuição das dificuldades e perigos encontrados significa a mudança dos padrões mais presentes no interior das residências (JUNIOR RC et al, 2013).

Um ambiente acessível é um fator facilitador para inúmeros idosos que apresentam limitações nas tarefas diárias ou não, com a introdução de projetos em design de interiores baseados em uma visão holística dos indivíduos envolvidos é possível diminuir déficits motores e favorecem o alcance da independência funcional para as atividades de vida diária com mais segurança, facilitando o deslocamento e ajudando a prevenir acidentes, especialmente as quedas (ALMEIDA RLS et al, 2015).

As características que devem permear o conhecimento dentro dos projetos e arquitetura e design de interiores devem focar não apenas em ambientes de convivência coletiva, mas principalmente os ambientes onde o idoso realiza atividades individuais, como quarto banheiro e salas de trabalho. Devido ao grande risco presente em ambientes pouco acessíveis as atividades do cotidiano acabam apresentando barreiras, e a capacidade funcional dessa população acaba limitando-se pela diminuição da capacidade de exercer atividades que possam aumentar o seu nível cognitivo e de força muscular, entre as inúmeras características que esse comportamento pode causar ainda é possível elencar como principais os riscos de queda, desconforto e até mesmo a insegurança, dessa maneira, torna-se essencial a realização de ajustes para o estímulo da autonomia e independência dos idosos (ALMEIDA RLS et al, 2015).

A responsabilidade sobre o direito de moradia digna não se relaciona apenas ao trabalho exercido por um profissional da arquitetura e/ou design de interiores, sabe-se que este é um direito relacionado aos direitos sociais e do ser humano. A implementação das políticas públicas habitacionais necessita ser efetivada no âmbito de proteção de direitos sociais e que possa ocorrer de forma democrática respeitando as diversidades do ambiente estrutural. Portanto, a moradia digna para o idoso de classe baixa, média ou alta, associada acessibilidade encontram guarida no Ministério Público, instituição adequada à concretização desse direito, podendo utilizar-se, dessa forma, do Termo de Ajustamento de Conduta (TAC) firmado em procedimentos extrajudiciais para garantir um direito inerente ao ser humano (SILVA NM et al, 2015).

A eliminação das barreiras arquitetônicas ainda é uma das melhores formas de possibilitar uma maior sensibilidade e redução do número de riscos de acidente no espaço doméstico. De acordo com o estudo de NEVES FC e BIFANO ACS (2015), para idosos entre 60 e 69 anos de idade os riscos que mais interferem na acessibilidade estão presentes em $80 \%$ das residências sendo que os mais encontrados estão no quintal e no banheiro representados pela presença de vasos e plantas, irregularidades no piso a falta de barras de apoio nos banheiros, tais fatores incrementam uma taxa de $45 \%$ de responsabilidade nos acidentes envolvendo queda, nesse sentido vale a pena ressaltar que a disposição não só da estrutura física, mas até mesmo do mobiliário é responsável em grande parte pelos acidentes no ambiente doméstico.

Atualmente os projetos em arquitetura e design de interiores podem habilitar ou inabilitar o usuário dentro do contexto de inclusão social e até mesmo podem ignorar conceitos de um desenho universal que muitas vezes são princípios básicos para tornar algo ou um lugar acessível à maioria dos indivíduos. A destreza científica e a usabilidade de defender as estruturas de adaptação e facilitação do dia-a-dia são necessárias para as barreiras em que os ambientes são construídos, para que possam refletir segurança e, acima de tudo, um ambiente agradável e ergonômico. Os acidentes envolvendo idosos ainda constituem um agravo frequente e multifatorial nos ambientes residenciais e vem despertando preocupações cada vez maiores entre os profissionais da arquitetura e do design de interiores, principalmente, no reconhecimento dos fatores de risco que levam um indivíduo a desenvolver a probabilidade de acidentes, assim como os meios eficazes de prevenção.

\section{CONSIDERAÇÕES FINAIS}

O atual cenário sócio demográfico do Brasil caracterizado, entre outros fatores, pelo envelhecimento populacional traz a consciência da necessidade de mecanismos que garantam melhorias a essa população. A ergonomia visando à otimização das condições de moradia, tem apresentado grandes avanços no que se 
refere a melhorias do ambiente, servindo como meio de conscientização de que o individuo exerce o papel central nesse processo e que o meio em que o mesmo se encontra deve ser adaptado ao homem, permitindo assim, a transformação do paradigma de adaptação do homem ao lar. Os projetos que visam a acessibilidade necessitam atribuir métodos e rotinas que possam de maneira eficaz reduzir e controlar qualquer alteração no espaço de vivência do idoso. Estima-se que o ambiente, o ritmo, modelo de disposição de móveis e até mesmo as interações humanas, principalmente da maneira como hoje são encontradas, possam gerar no idoso um elevado desgaste cognitivo físico e afetivo para a execução das atividades cotidianas.

\section{REFERÊNCIAS}

1. ALMEIDA RLS, et al. Instituição de longa permanência para idosos: avaliação das condições de acessibilidade e da funcionalidade dos idosos. Rev. Saúde. Com., 2015; (11)2: 162-173.

2. AUSTREGÉSILO SC, et al. Acessibilidade a serviços de saúde bucal por pessoas idosas: uma revisão integrativa. Rev. Bras. Geriatr. Gerontol., Rio de Janeiro, 2015; 18(1): 189-199.

3. FERNANDES JCFA, CARVALHO RJM. Mapeamento da acessibilidade nas instituições de longa permanência para idosos da cidade de natal-rn. XXXI Encontro Nacional de Engenharia de Producão, 2011.

4. GARBIN CAS, et al. Histórico de quedas e acessibilidade do idoso em instituições de longa permanência. Arch Health Invest, 2015; 4(4): 29-38.

5. GIRONDI JBR, SANTOS SMA. Deficiência física em idosos e acessibilidade na atenção básica em saúde: revisão integrativa da literatura. Rev Gaúcha Enferm., Porto Alegre, 2011; 32(2): 378-384.

6. JÚNIOR RCF, et al. Estudo da acessibilidade de idosos ao centro da cidade de Caratinga, MG. Rev. Bras. Geriatr. Gerontol., Rio de Janeiro, 2013; 16(3): 541-558.

7. LUIZ FI, TEIXEIRA JC. A acessibilidade de idosos e as unidades de conservação: reflexões rumo à democratização dos espaços públicos de lazer. Revista Turismo - Visão e Ação - Eletrônica, 2016; 18(1): 164-192.

8. NEVES FC, BIFANO ACS. O processo de envelhecimento e acessibilidade: o idoso no espaço domiciliar. Anais CIEH, 2015; 2(1).

9. SANTOS MIPO, et al. Acessibilidade e acolhimento: estratégias potenciais para qualificação da assistência à saúde do idoso. Rev. Bras. Pesq. Saúde, Vitória, 2016; 18(2): 42-51.

10. SILVA NM, et al. Necessidades próprias da (c) idade: espaços acessíveis e funcionais para idosos. Serv. Soc. Rev., Londrina, 2015; 18(1): 219-242.

11. TOWATA TM. Análise da iluminação e acessibilidade de instituições de longa permanência de idosos em Campo Grande, MS. Revista Especialize On-line IPOG - Goiânia, 2014; 9(1): 1-19. 